\title{
Modeling the Distribution Characteristics of Urban Public Bicycle Rental Duration
}

\author{
Shuichao Zhang, Kangkang He, Sheng Dong, and Jibiao Zhou \\ School of Transportation, Ningbo University of Technology, Ningbo 315211, China \\ Correspondence should be addressed to Shuichao Zhang; zhangsc2588509@126.com
}

Received 1 September 2015; Revised 11 November 2015; Accepted 19 January 2016

Academic Editor: Aura Reggiani

Copyright ( 2016 Shuichao Zhang et al. This is an open access article distributed under the Creative Commons Attribution License, which permits unrestricted use, distribution, and reproduction in any medium, provided the original work is properly cited.

\begin{abstract}
In order to model the distribution characteristics of public bicycle rental durations, individual journey data for three cities in China (Ningbo, Hangzhou, and Beijing), for weekdays, was obtained. The distribution curves for public bicycle rental duration in the three cities were found to be extremely similar, with small differences among the weekdays. The basic parameters such as the average rental duration, the rental duration corresponding to the maximum rental frequency, and the rental duration corresponding to $75 \%$ degree were then calculated. On this basis, the radioactive decay law from physics was used to establish a theoretical model for the relationship between rental frequency and rental duration. The data on public bicycle rental duration in Ningbo, Hangzhou, and Beijing were used to test the model and produce a corrected theoretical model. The results indicate that the relationship between rental frequency and rental duration obeys the decay law. The study results provide important theoretical support for the rental station planning of bicycle sharing systems, as well as the allocation, operation, and dispatch of public bicycles.
\end{abstract}

\section{Introduction}

Compared to private bicycles, public bicycles are well received by Chinese people, who need not fear that their bicycles will be stolen or that they will not have space to park. People who use public bicycles also do not need to spend time repairing their bicycles themselves [1]. In addition, the Chinese government strongly advocates travel by public transportation and has therefore spent heavily on the construction of a bicycle sharing system in order to ease urban traffic congestion. The Chinese bicycle sharing system has developed rapidly, with 123 cities having adopted bicycle sharing systems within just a few years, for a total of 510,000 bicycles and 20,000 rental stations available. These cities include metropolises such as Beijing and Shanghai, large cities such as Hangzhou and Nanjing, and medium-sized cities such as Ningbo and Suzhou [2], where the bicycle sharing system has been completely constructed and operates stably. This growth has led China to become one of the major areas worldwide in which public bicycles are broadly distributed [3].

However, as a new emerging public transit network, the bicycle sharing systems in most cities currently face problems that significantly affect their overall benefits, including station placement, stand allocation, and bicycle dispatch. A lack of relevant basic theory presents another major issue that must be solved during the development of the public bicycle sharing system. Rental duration as studied in this paper refers to the period of time that a rider spends in cycling from the departure station to the destination station; it reflects the temporal and spatial cycling distance between the two stations. Through theoretical modeling and actual data testing, this paper studies the distribution characteristics of rental duration, which refer to the frequencies of different rental duration and generally reflect riders' preferences with regard to cycling time or cycling distance, as well as their general desire for cycling. This study can provide theoretical support for predicting demand for public bicycles, bicycle station planning, allocation of stands at bicycle stations, and the operation and dispatch of public bicycles, matters that hold both theoretical and practical significance.

Domestic and foreign studies on the distribution characteristics of public bicycle rental duration have been carried out from diverse perspectives and have yielded some notable results. For example, by studying the bicycle sharing system in Suzhou, Zhou et al. analyzed the characteristics associated with the use of public bicycles as a means of transfer 
between subways based on data obtained from riders' cards and questionnaires. Zhou et al. pointed out that the rental duration of public bicycles at stations specially designated for subways was short and discrete, and they differed significantly from those of public bicycles at other types of stations [4]. Using research on the operation data of Lyon's public bicycle system, Jensen et al. found that the average travel distance for a public bicycle was $2.49 \mathrm{~km}$, and the average travel time was less than $15 \mathrm{~min}$ [5]. An extensive investigation conducted by Shaheen et al. revealed that users of bicycle sharing systems in North America generally live nearer to their workplace than the general population and that travel distance is a major factor influencing residents' choice to use public bicycles [6]. Joo et al. developed a methodology for categorizing bicycling environments according to the bicyclist's perceived level of safety and comfort. In this study, second-by-second bicycle speed data were collected using global positioning systems (GPS) on public bicycles, and a set of features characterizing the bicycling environments was extracted from the GPSbased bicycle speed and acceleration data [7].

Recent studies on bicycle sharing systems have mostly presented results in terms of construction and planning, operational management, and the layout and planning of public bicycle rental stations. For example, He et al. analyzed the characteristics and problems associated with the current use of public bicycles, established an optimization model for the layout of urban public bicycle rental stations, and validated the model using a real-life example [8]. Based on data obtained from riders' cards and questionnaires, Zhu et al. summarized the operation characteristics of public bicycle stations for different regions and different types of land use and offered advice for the planning of public bicycle stations [9]. Some scholars suggest paying attention to the construction of urban bicycle lanes in order to improve the efficiency of bicycle sharing systems. For example, based on investigations of the distribution and operation of public bicycle stations in Hangzhou's urban center, business districts, and residential areas, Qian et al. [10] pointed out that development must focus on station placement and the construction of bicycle lanes. In terms of operational management, Liege et al. studied the imbalanced demand for urban bicycle sharing systems during peak hours, discussed the optimization of public bicycle scheduling, and constructed a model for optimal vehicle scheduling [11]. By studying people's means of travel in Melbourne, Lansell identified frequent transfers between public bicycles and rail transportation, a phenomenon that is especially pronounced during traffic peak hours. He also found that more than half of the respondents combined public bicycles with the rail transit system [12]. Based on relevant data about the public bicycle rental system in Helsinki, Finland, Jäppinen et al. simulated the overall operation and dispatch of the bicycle sharing system and suggested that public bicycles can greatly improve the accessibility of an urban transportation system [13].

Traffic systems have widely used physical models such as the gravity model and the principle of maximum entropy $[14,15]$, but physical modeling of bicycle sharing systems has occurred less frequently during research studies. By analyzing data about public bicycle rentals in thirty-eight cities in Europe, the Middle East, Asia, Australia, and the United States, O'Brien et al. obtained basic characteristics of vehicle occupancy at public bicycle rental stations. He proposed that public bicycles can be modeled and analyzed as a relatively closed system [16]. However, studies on bicycle sharing systems using physical modeling have yielded few results, and the radioactive decay law has almost never been applied to the problem. So far, modeling of bicycle sharing systems has primarily been mathematical in nature. For example, Wu et al. established a demand prediction model for public bicycle rentals based on the multinomial logit model [17]. Castillo-Manzano et al. applied a discrete choice model after fieldwork based on surveys conducted in Seville, Spain; the findings enumerated sociodemographic factors that favor the move from the public bicycle to the private bicycle, including having a higher level of education, being more ideologically progressive, and being a resident of the city itself [18].

In view of the lack of studies on the distribution characteristics of public bicycle rental duration, this paper will provide a physical model based on actual data about the characteristics of public bicycle rental duration. The results can provide a theoretical basis for the planning and operation of bicycle sharing systems.

\section{Data Acquisition}

Currently, bicycle sharing systems have been completely implemented in metropolises, large cities, and medium-sized cities in China. Therefore, Ningbo, Hangzhou, and Beijing were selected to represent these three categories of cities. Table 1 provides basic information about the three target cities.

Based on research needs, data on public bicycle rentals were obtained from IC cards in 2014 for six weekdays in Ningbo, five weekdays in Hangzhou, and five weekdays in Beijing. The researchers obtained the data by contacting the appropriate departments for the bicycle sharing systems in these cities. The targeted weekdays were all clear days suitable for cycling. The following serves as an introduction to the data obtained from Ningbo and the method by which the data were processed.

Currently, IC cards are used to rent and return bicycles in China's bicycle sharing systems. When a lessee completes a rental, the system records the number of the bicycle rented by the lessee, the station and stand from which the bicycle is rented, the station and stand to which the bicycle is returned, and the times at which the bicycle is rented and returned. Thus, the basic rental information for public bicycles can be obtained by exporting the system's background database. By contacting the public bicycle management companies in Beijing, Hangzhou, and Ningbo, we obtained access to the databases containing public bicycle rental information, and we retrieved information for six weekdays in Ningbo, five weekdays in Hangzhou, and five weekdays in Beijing. The systems yielded data about 90,000 daily rentals, 170,000 daily rentals, and 210,000 daily rentals, respectively, and each datum contained the lessee's card number, the number of the bicycle rented, the station and stand from which the bicycle 
TABLE 1: Basic information about the three cities.

\begin{tabular}{|c|c|c|c|}
\hline City name & Ningbo & Hangzhou & Beijing \\
\hline City type & Medium-sized city & Large city & Metropolis \\
\hline $\begin{array}{l}\text { Date of bicycle sharing system } \\
\text { construction }\end{array}$ & 2012 & 2007 & 2008 \\
\hline Primary area of distribution & Downtown & Downtown & Core \\
\hline Area of coverage & About $145 \mathrm{~km}^{2}$ & About $185 \mathrm{~km}^{2}$ & About $250 \mathrm{~km}^{2}$ \\
\hline Station density & 8.6 stations per $\mathrm{km}^{2}$ & 9.2 stations per $\mathrm{km}^{2}$ & 8.9 stations per $\mathrm{km}^{2}$ \\
\hline Bicycle lanes & $\begin{array}{l}\text { Independent, connected bicycle } \\
\text { lanes on urban roads }\end{array}$ & $\begin{array}{l}\text { Independent, connected bicycle } \\
\text { lanes on urban roads }\end{array}$ & $\begin{array}{l}\text { Independent, connected bicycle } \\
\text { lanes on urban roads }\end{array}$ \\
\hline $\begin{array}{l}\text { Number of public bicycles in the } \\
\text { area of primary distributed }\end{array}$ & About 25,000 bicycles & About 40,000 bicycles & About 50,000 bicycles \\
\hline Operation $^{*}$ & Government-operated & Government-operated & Government-operated \\
\hline Rental method & IC cards & IC cards & IC cards \\
\hline Charging standards & $\begin{array}{l}\text { Available free of charge for } \\
\text { rentals of less than one hour; } \\
\text { after one hour, charged on an } \\
\text { hourly basis }\end{array}$ & $\begin{array}{l}\text { Available free of charge for } \\
\text { rentals of less than one hour; } \\
\text { after one hour, charged on an } \\
\text { hourly basis }\end{array}$ & $\begin{array}{l}\text { Available free of charge for } \\
\text { rentals of less than one hour; } \\
\text { after one hour, charged on an } \\
\text { hourly basis }\end{array}$ \\
\hline
\end{tabular}

* Note: almost all bicycle sharing systems are operated by the local government in Chinese cities.

was rented, the station and stand to which the bicycle was returned, and the times at which the bicycle was rented and returned. The duration of each rental can be obtained based on this information. Table 2 shows examples of the basic data for Ningbo.

Since the rental information databases for the three cities were generally the same, a detailed introduction is only given for the method of processing the Ningbo data. Two types of entries were eliminated from the datasets: data corresponding to the rental duration of zero and data corresponding to the rental duration of over one hour. The rental duration of zero indicates that the lessee did not travel; this type of data accounted for about $1.1 \%$ of the total data. Data corresponding to a rental duration exceeding one hour were eliminated in order to ensure the convergence and oneness of data. For one thing, public bicycles are manually operated, and continuous cycling in excess of one hour is rare, accounting for about $0.8 \%$ of the total data; additionally, renting a public bicycle for less than an hour is free of charge in China, and payment is only required for public bicycle rentals exceeding one hour. In all, 80,000 to 90,000 valid data points were obtained for each of the weekdays in Ningbo. Data from the other two cities were processed in a similar way.

\section{Rental Duration Distribution Characteristics}

For ease of study and analysis, the frequencies of rental duration for Ningbo were collected in units of minutes; for example, the label "five minutes" is used to express the period of time from five minutes, zero seconds, to five minutes, fifty-nine seconds. The frequency data collected for the six weekdays and their averages were tabulated to yield the frequency distributions of different rental duration, as shown in Figure 1.

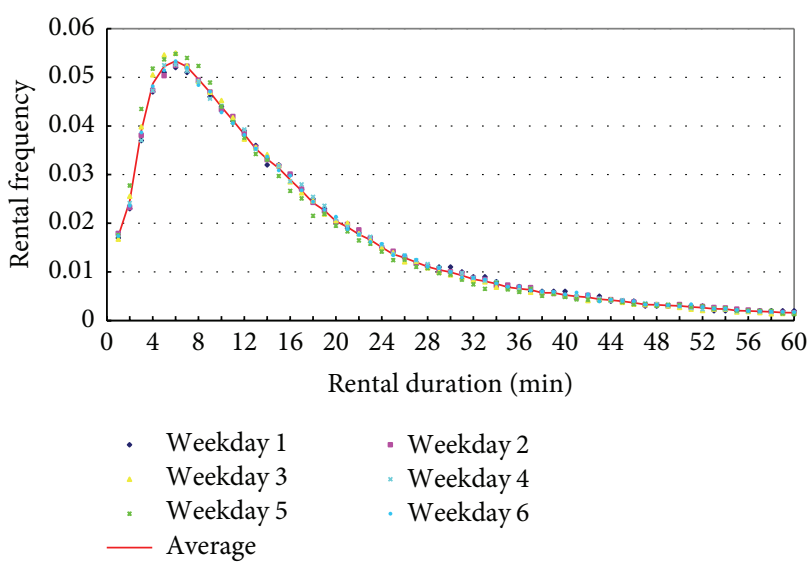

FIGURE 1: Frequency distributions of public bicycle rental duration in Ningbo.

Figure 1 shows an obvious peak in the frequency distribution curves of public bicycle rental duration. The curve is cliff-like to the left of the peak and gentler than the right of the peak. Overall, the differences among the weekdays' data and their average are small.

The data obtained from Beijing and Hangzhou was compared with the data from Ningbo, yielding Figure 2 and Table 3.

Table 3 shows that the distribution characteristics of public bicycle rental duration were extremely similar across the three cities, with their averages being about 15 minutes and frequency distribution peaks occurring at 6 minutes. In Ningbo, 25\% of cycling trips took less than 6 minutes, and $75 \%$ took less than 21 minutes, indicating that public bicycles are used for short-distance travel and that people prefer other means of transportation when they travel long distances. 


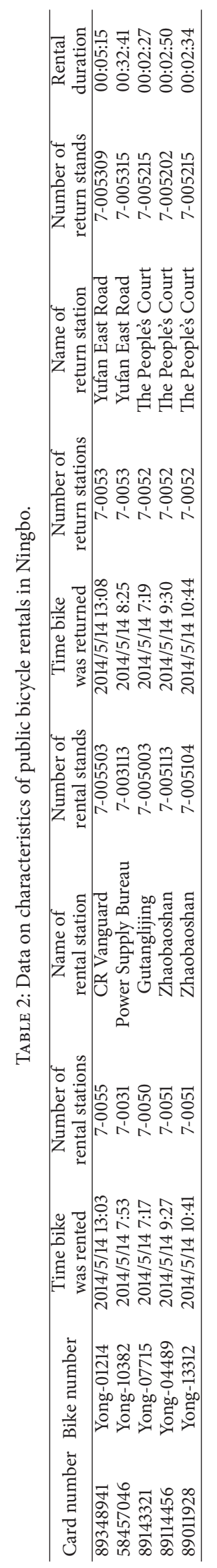


TABLE 3: Characteristics of public bicycle rental duration for the three cities.

\begin{tabular}{lccccc}
\hline \multirow{2}{*}{ City } & \multirow{2}{*}{ Average rental duration } & \multicolumn{2}{c}{ Distribution peak } & \multicolumn{3}{c}{ Percentile of cumulative frequency } \\
& & Rental duration & Rental frequency & $25 \%$ & $50 \%$ \\
\hline Ningbo & 15.88 & 6 & 0.0536 & 6 & 12 \\
Beijing & 14.78 & 6 & 0.0560 & - & - \\
Hangzhou & 15.12 & 6 & 0.0548 & - & - \\
\hline
\end{tabular}

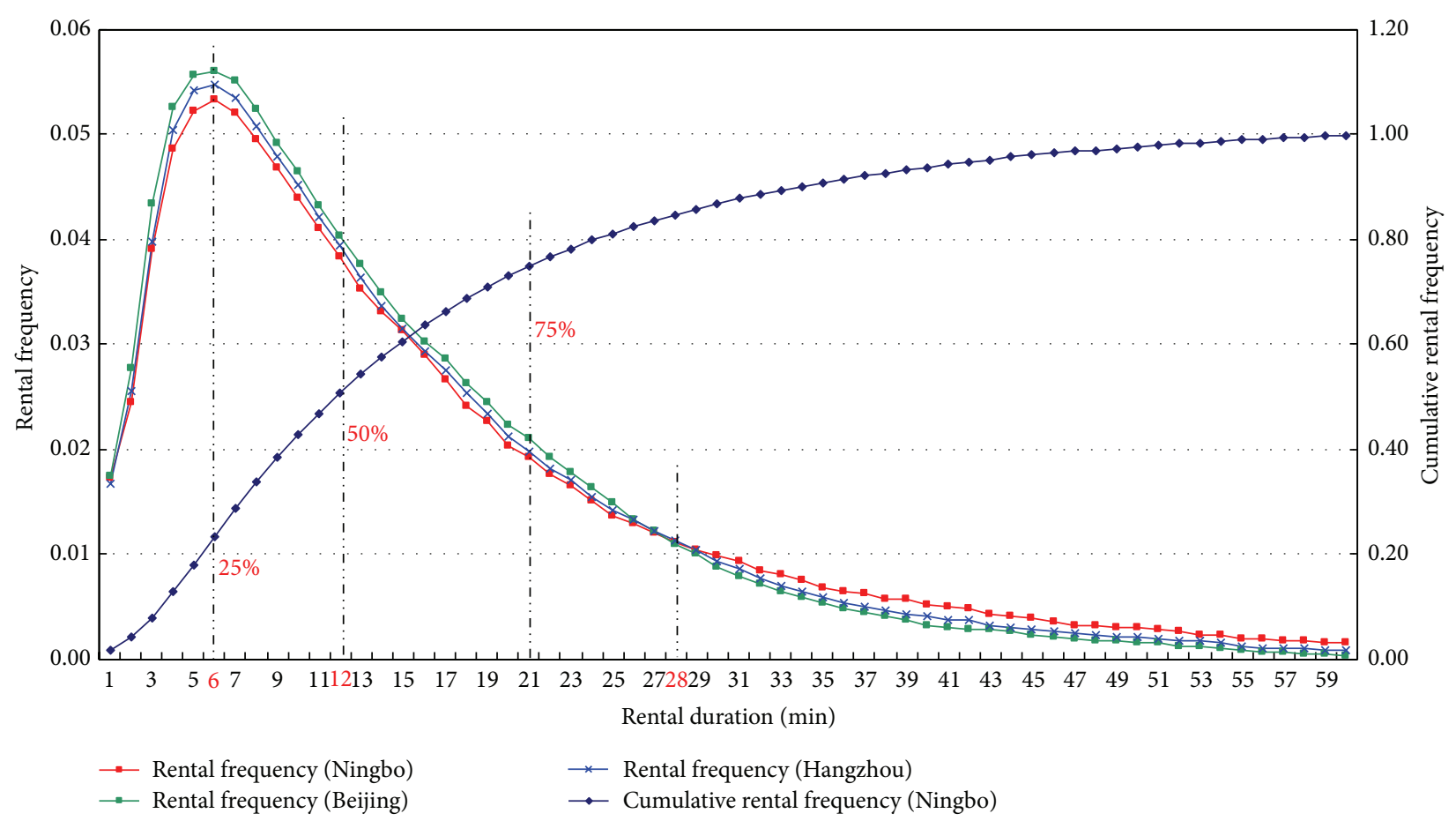

FIGURE 2: Characteristics of public bicycle rental duration for the three cities.

Figure 2 shows that the frequency of rental duration shorter than 28 minutes was highest for Beijing and lowest for Ningbo, and the frequency of rental duration longer than 28 minutes was lowest for Beijing and highest for Ningbo. This may be because the public transportation systems in Beijing and Hangzhou are more developed than that in Ningbo, such that people who use public bicycles in the former two cities are able to use other forms of public transportation when traveling longer distances.

Rental data for three weekends were obtained from Ningbo in order to expand the application of the study results of this paper, and the average data for the weekends were compared to the weekday data. Figure 2 shows the results.

As Figure 3 shows, the rental duration distribution characteristics for weekends are extremely similar to those for weekdays, indicating that rental duration distribution characteristics are basic characteristics of bicycle sharing systems. However, the obtained data revealed that the number of weekend rentals was significantly smaller than the figures for weekdays. About 100,000 daily rentals occurred on weekdays, and only about 50,000 daily rentals occurred on weekends.

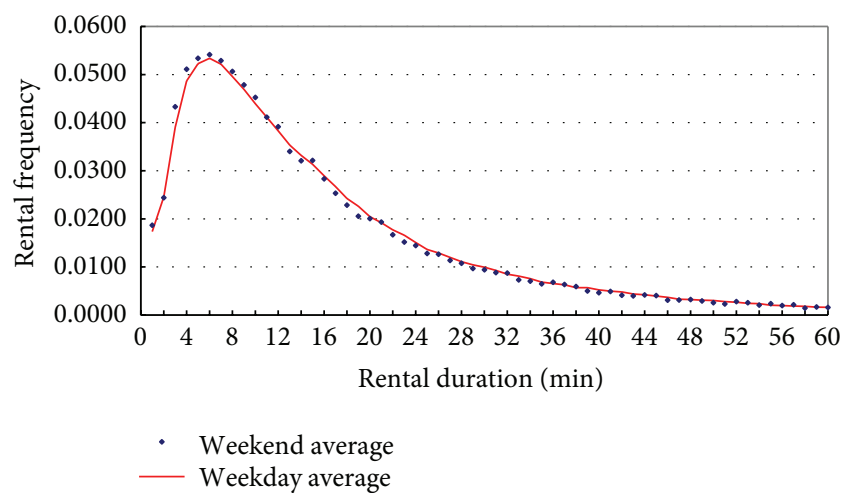

FIGURE 3: Comparison of rental duration distribution characteristics for weekdays and weekends.

\section{Modeling}

4.1. Basic Assumptions. The rental duration of a public bicycle is the temporal cycling distance between the departure station 
and the destination station, and it generally reflects the spatial cycling distance. Since bicycles are manually driven, people exert more physical effort as cycling continues, and the advantages of cycling gradually weaken. According to this analysis, a person's desire to rent a bicycle to travel between two stations should be inversely proportional to the rental duration between two stations.

Based on the above analysis and the frequency distributions of public bicycle rental duration shown in Figure 2, the radioactive decay law from physics was used to establish a relationship between a person's desire to rent a bicycle to travel between two stations and the rental duration, that is, the temporal distance between the two stations. The process by which the nucleus of a radioactive element changes into a different element's nucleus due to loss of a certain particle is called the decay of a radioactive element, and this process complies with the decay law. The decay law states that the rate at which the number of the nuclei of a radioactive element decays or decreases is proportional to the number of the remaining nuclei; namely, $[19,20]$

$$
\frac{d N(t)}{d t}=-k N(t)
$$

In (1), named subsequently as radiation model, $N(t)$ is the number of the remaining nuclei at a certain moment of time, $d N(t) / d t$ is the rate of decay of the nuclei, and $k$ is a decay constant that represents the rate of decay of a radioactive element.

Interestingly, (1) is, under certain assumptions, the dynamic version of the conventional spatial interaction model, embedding an exponential decay function, which is conventionally used in transport and spatial economic science. For applications of spatial interaction modeling to transport flows see [19-21].

Let $D(T)$ represent a person's desire to rent a bicycle to travel between two stations whose temporal distance is $T$. If the temporal distance between these two stations increases by $d T$, then the person's desire to rent a bicycle will show a decrease gradient of $d D(T) / d T$. This paper assumes that according to the decay law the decrease gradient is proportional to the current desire to rent a bicycle; namely,

$$
\begin{gathered}
\frac{d D(T)}{d T}=-k D(T), \\
D(T)=C_{1} e^{-k T} .
\end{gathered}
$$

In these equations, $C_{1}$ and $k$ are parameters to be determined. In traffic engineering, $k$ is defined as the sensitivity of a person's desire to rent a bicycle to travel a certain duration or distance. Table 4 illustrates the relationship between public bicycle system and radiation model.

4.2. Modeling. Generally speaking, the number of rentals between two stations should be proportional to travelers' desire to rent. In other words, the number of actual rentals $f(T)$ between a station and another station at a temporal
TABLE 4: The relationship between public bicycle system and radiation model.

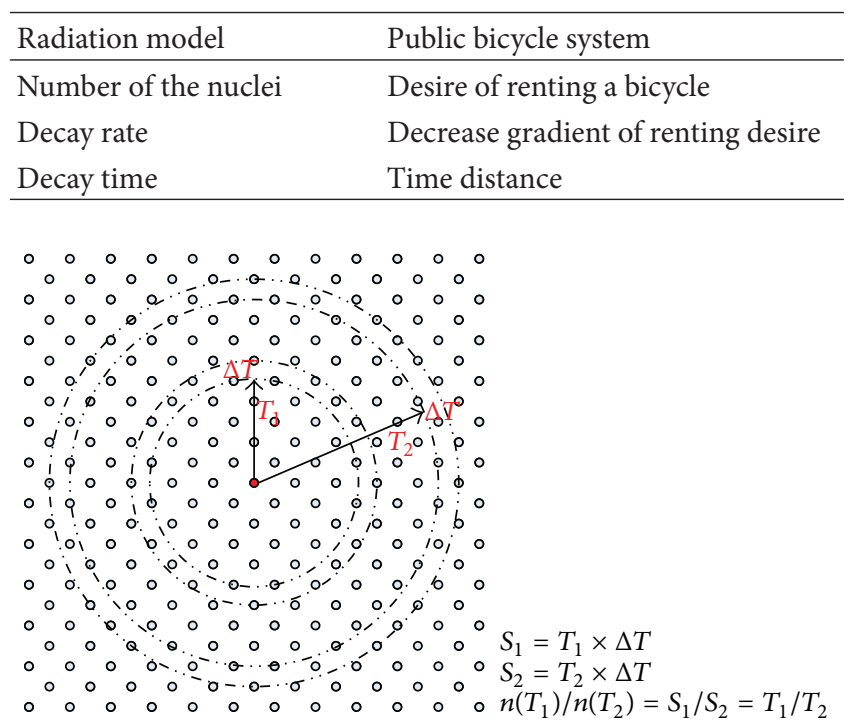

FIGURE 4: Analysis of the proportional relation of the number of pairs of stations $n(T)$ and $T$.

distance of $T$ should be proportional to the desire for rentals between the two stations, $D(T)$; namely,

$$
f(T)=C_{2} D(T) .
$$

In this equation, $C_{2}$ is the parameter to be determined. The number of rentals with duration $T$ in a city is also proportional to the number of matching stations $n(T)$ whose temporal distance is $T$; from another perspective, this can be understood as the satisfaction of actual demand for rentals being constrained by the number of rental stations. Figure 4 illustrates that if the stations in a city are evenly placed, then $n(T)$ is also proportional to $T$. Therefore,

$$
F(T)=C f(T) n(T)=C T e^{-k T} .
$$

In this equation, $C$ and $k$ are parameters to be determined. The number of rentals $F(T)$ in a city is large and is usually expressed as frequency.

In Figure 4, the dots represent the stations, and the red dot in the center represents the benchmark station. The stations are evenly placed. $S_{1}$ represents the area of the zone between the circles with the radii of $T_{1}$ and $T_{1}+\Delta T . \Delta T$ is a small amount and $n\left(T_{1}\right)$ is the number of stations in the zone. So, $n\left(T_{1}\right)$ represents the number of the stations whose time distance from the benchmark station is $T_{1}$. Because the stations are evenly placed, $n\left(T_{1}\right)$ is proportional to $S_{1} . T_{1}, S_{2}$, and $n\left(T_{2}\right)$ have the same meanings.

\section{Model Testing}

It can be seen from Figure 2 or Table 3 that the rental frequency curve of Ningbo has only one extreme point, located at $(6,0.0536)$, and the extreme point is also the only 
TABLE 5: Comparison of the functions of the three cities' public bicycle rental duration models.

\begin{tabular}{|c|c|c|c|c|c|c|c|c|c|}
\hline City & & Ningbo & & & Beijing & & & Hangzhou & \\
\hline Model function & $F(T)=$ & $\left\{\begin{array}{l}0.0243 T e^{-0.1667 T}, \\
0.0706 e^{-0.0641 T},\end{array}\right.$ & $\begin{array}{l}T \leq 17 \\
T>17\end{array}$ & $F(T)=$ & $\left\{\begin{array}{l}0.0254 T e^{-0.1667 T}, \\
0.1558 e^{-0.0948 T},\end{array}\right.$ & $\begin{array}{l}T \leq 17 \\
T>17\end{array}$ & $F(T)=$ & $\left\{\begin{array}{l}0.0248 T e^{-0.1667 T}, \\
0.0994 e^{-0.0784 T},\end{array}\right.$ & $\begin{array}{l}T \leq 17 \\
T>17\end{array}$ \\
\hline
\end{tabular}

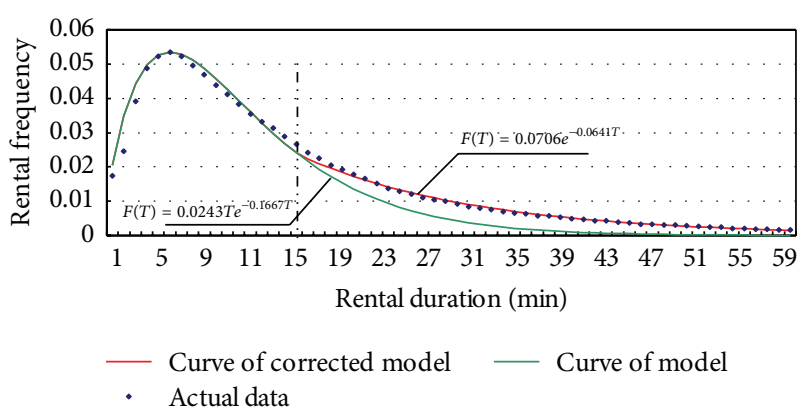

FIgURE 5: Data fitting.

one extreme point of $F(T)$. Therefore, $F(T)$ is subject to the following two constraints:

$$
\begin{aligned}
& F^{\prime}(6)=0, \\
& F(6)=0.0536 .
\end{aligned}
$$

Therefore, $C=0.0243$, and $k=0.1667$; namely,

$$
F(T)=0.0243 T e^{-0.1667 T} .
$$

Figure 5 depicts the curve for this function or model, as well as the actual data. When $T \leq 17$, the curve is highly consistent with the actual data, but when $T>17$, the two are less consistent.

In order to study the distribution characteristics of rental duration further, the actual data where $T$ was greater than 17 were fitted separately. It was found that these data generally obey $F(T)=0.0706 e^{-0.0641 T}$, as shown in Figure 4 by the curve of corrected model. Based on the type of function, it can be stated that when $T>17$, the satisfaction of public bicycle rental demand is no longer restricted by the number of stations, due to the decrease in demand for rentals and the significant increase in the number of matching stations. The function generally obeys the decay law. The data for Beijing and Hangzhou were also fitted, and functions were obtained for the rental duration models for all three cities, as shown in Table 5.

As Table 4 illustrates, the distribution characteristics of public bicycle rental duration for Ningbo, Beijing, and Hangzhou generally obey the decay law. Therefore, the model established in this paper may be able to describe the distribution characteristics of public bicycle rental duration. Equation (4) was properly corrected to yield

$$
F(T)= \begin{cases}C_{1} T e^{-k_{1} T}, & T \leq T_{0} \\ C_{2} e^{-k_{2} T}, & T>T_{0}\end{cases}
$$

in which $C_{1}, C_{2}, k_{1}, k_{2}$, and $T_{0}$ are parameters to be determined.

\section{Discussion}

Based on this study, the following aspects emerge for discussion:

(1) Based on data about public bicycle rental duration for Ningbo, Beijing, and Hangzhou, the distribution characteristics of rental duration for the three cities are extremely similar. This is because of the fundamental characteristics of bicycles, which are manually driven, with a cycling speed generally maintained at about $14 \mathrm{~km} / \mathrm{h}$; therefore, the option of traveling on public bicycles is primarily affected by the amount of physical strength and time required, which differ a little among people in different cities. This study calculated the average public bicycle rental duration to be about 15 minutes, which is fundamentally consistent with the conclusion drawn by Jensen et al. that the average rental duration of public bicycles in Lyon was less than 15 minutes [5]. This indicates that Chinese cities may differ slightly from cities in other countries in the basic characteristics of public bicycle rentals; however, due to a lack of relevant study results, the parameters for the distribution characteristics of public bicycle rental duration obtained in this paper cannot be compared with data from cities in other countries.

(2) According to the function for the corrected model expressed by (7) and the instance parameters in Table $4, k_{1}=0.1667$, and $k_{2}=0.0641$. In the model established in this paper, $k$ represents public bicycle riders' sensitivity to rental duration. For longdistance travel, public bicycles lose their advantages, and other factors such as exercise, environmental protection, and undeveloped public transportation more strongly affect people's decisions to travel on public bicycles; therefore, $k_{2}<k_{1}$ means that public bicycle riders' sensitivity to rental duration declines when $T>T_{0}$. The value of $k_{2}$ was smallest for Ningbo and greatest for Beijing, which is consistent with the above analysis.

(3) In the corrected model, $T_{0}$ is the intersection of the two subfunctions. However, the actual data interval between $T_{0}-2$ and $T_{0}+2$ was found to be less consistent with the two subfunctions than other intervals. Therefore, this interval can be considered a transition between the two subfunctions that is affected by the two subfunctions. 
TABLE 6: Applications of the study results.

\begin{tabular}{|c|c|}
\hline Applications & Methods for applying results \\
\hline $\begin{array}{l}\text { Planning of public bicycle } \\
\text { rental stations }\end{array}$ & $\begin{array}{l}\text { Use the basic parameter such as the } \\
\text { average rental duration, the rental } \\
\text { duration corresponding to the } \\
\text { maximum rental frequency, and the } \\
\text { rental duration corresponding to } 75 \% \\
\text { degree to determine a reasonable } \\
\text { rental station interval. This will } \\
\text { provide a basis for the planning of } \\
\text { rental stations. }\end{array}$ \\
\hline $\begin{array}{l}\text { Determination of the } \\
\text { number of bicycles } \\
\text { allocated at stations }\end{array}$ & $\begin{array}{l}\text { Use the concrete functional } \\
\text { expression of the distribution } \\
\text { characteristics to predict the } \\
\text { origin-destination (OD) demand } \\
\text { between public bicycle rental } \\
\text { stations. This will provide a basis for } \\
\text { the allocation of public bicycles at } \\
\text { rental stations. }\end{array}$ \\
\hline Public bicycle scheduling & $\begin{array}{l}\text { Use the functional expression of the } \\
\text { distribution characteristics of rental } \\
\text { duration to establish a model that } \\
\text { simulates the movement of public } \\
\text { bicycles between stations and studies } \\
\text { the dynamic scheduling of bicycle } \\
\text { sharing systems. }\end{array}$ \\
\hline
\end{tabular}

(4) The distribution characteristics of public bicycle rental frequencies versus rental duration, as obtained in this study, reflect the distribution characteristics of the rental demand of public bicycle lessees versus rental duration. Therefore, the basic parameters directly reflected in the distribution characteristics are very practical, and they include the average rental duration, the rental duration corresponding to the maximum rental frequency, and the rental duration corresponding to $75 \%$ degree were then calculated. The concrete functional expression of the distribution characteristics is also very practical. Table 6 illustrates the applications of this study's results for the planning of public bicycle rental stations, the determination of the number of public bicycles allocated at public bicycle stations, and public bicycle scheduling.

\section{Conclusions}

The distribution of public bicycle rental duration, which is an important fundamental characteristic of a bicycle sharing system, was assumed in this paper to be subject to modeling using the radioactive decay law from physics. Through actual data testing, the model proved to be well capable of describing the distribution characteristics of public bicycle rental duration, and it was established in the form of a piecewise function. The study results of this paper promise wide applications by providing a solid theoretical basis for locating public bicycle rental stations, allocating bicycles at bicycle stations, and dispatching of public bicycles.

\section{Conflict of Interests}

The authors declare that there is no conflict of interests regarding the publication of this paper.

\section{Acknowledgments}

This research is funded by the National Natural Science Foundation of China (51308311), the Natural Science Foundation of Zhejiang Province of China (LQ13E080004), the Social Science Foundation of Zhejiang Province of China (no. 15NDJC078YB), and the Natural Science Foundation of Ningbo City of China (no. 2015A610298). The authors hereby express their gratitude.

\section{References}

[1] D. Gong and Z. Zhu, "Implementation mechanism of urban public bicycle systems," Urban Transport of China, vol. 6, no. 6, pp. 27-32, 2008.

[2] D. Hang, "Current situation and countermeasures of Urban Public bicycle sharing system," Traffic \& Transportation, vol. 10, no. 1, pp. 30-31, 2015.

[3] Y. Zhou, "Urban bicycle sharing system planning," Urban Transport of China, vol. 10, no. 5, pp. 50-54, 2012.

[4] Q. Zhou, G. Wu, and H. Sun, "Characteristics of public bicycle as means of access/egress for metro," Journal of Transportation Systems Engineering and Information Technology, vol. 15, no. 3, pp. 179-184, 2015.

[5] P. Jensen, J.-B. Rouquier, N. Ovtracht, and C. Robardet, "Characterizing the speed and paths of shared bicycle use in Lyon," Transportation Research Part D: Transport and Environment, vol. 15 , no. 8, pp. 522-524, 2010.

[6] S. Shaheen, E. Martin, A. P. Cohen, and R. Finson, "Public bikesharing in north america: early operator and user understanding," Tech. Rep., Mineta Transportation Institute, San Jose, Cali, USA, 2012.

[7] S. Joo, C. Oh, E. Jeong, and G. Lee, "Categorizing bicycling environments using GPS-based public bicycle speed data," Transportation Research Part C: Emerging Technologies, vol. 56, pp. 239-250, 2015.

[8] L. He, D. Chen, X. Li, and J. Lu, "An optimization model of the layout of public bike rental stations," Journal of Wuhan University of Technology, vol. 36, no. 1, pp. 129-133, 2012.

[9] W. Zhu, Y. Pang, D. Wang, and X. Yu, "Research on the travel characteristics of public bicycle in minhang district, Shanghai," Urban Planning Forum, vol. 24, no. 5, pp. 76-81, 2012.

[10] J. Qian, Z. Zheng, and Y. Feng, "An assessment of the public bicycle facilities in Hangzhou," Planner, vol. 26, no. 1, pp. 71-76, 2010.

[11] H. Liege, Y. Xia, J. Wang, and L. Tang, "Scheduling optimization research on demand imbalance of urban public bicycles during peak period," Journal of Railway Science and Engineering, vol. 12, no. 2, pp. 441-448, 2015.

[12] K. Lansell, Melbourne bike share and public transport integration [M.S. thesis], University of Melbourne, Melbourne, Australia, 2011.

[13] S. Jäppinen, T. Toivonen, and M. Salonen, "Modelling the potential effect of shared bicycles on public transport travel times in Greater Helsinki: an open data approach," Applied Geography, vol. 43, pp. 13-24, 2013. 
[14] R. Chen, "Tri-constrained gravity model and its application," Urban Transport of China, vol. 7, no. 2, pp. 74-80, 2009.

[15] Y. Shao, L. Cheng, and W. Wang, "Application of EntropyMaximizing (EM) model in traffic distribution forecast," Journal of Transportation Systems Engineering and Information Technology, vol. 5, no. 1, pp. 83-87, 2005.

[16] O. O'Brien, J. Cheshire, and M. Batty, "Mining bicycle sharing data for generating insights into sustainable transport systems," Journal of Transport Geography, vol. 34, pp. 262-273, 2014.

[17] Y. Wu, H. Chen, N. Bao, and W. Feng, "A model development of public bicycle rental demand based on multinomial logit," Journal of Dalian Jiaotong University, vol. 34, no. 1, pp. 19-24, 2013.

[18] J. I. Castillo-Manzano, M. Castro-Nuño, and L. LópezValpuesta, "Analyzing the transition from a public bicycle system to bicycle ownership: a complex relationship," Transportation Research Part D: Transport and Environment, vol. 38, pp. 15-26, 2015.

[19] S. A. Fotheringham and M. E. O'Kelly, Spatial Interaction Models: Formulations and Applications, Kluwer Academic publishers, New York, NY, USA, 1989.

[20] A. G. Wilson, Geography and the Environment: Systems Analytical Methods, John Wiley \& Sons, New York, NY, USA, 1981.

[21] A. Reggiani, P. Bucci, and G. Russo, "Accessibility and impedance forms: empirical applications to the German commuting network," International Regional Science Review, vol. 34, no. 2, pp. 230-252, 2011. 


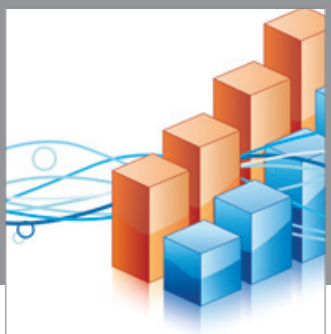

Advances in

Operations Research

vatem alat4

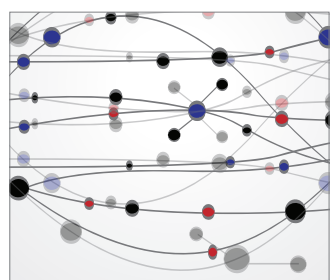

\section{The Scientific} World Journal
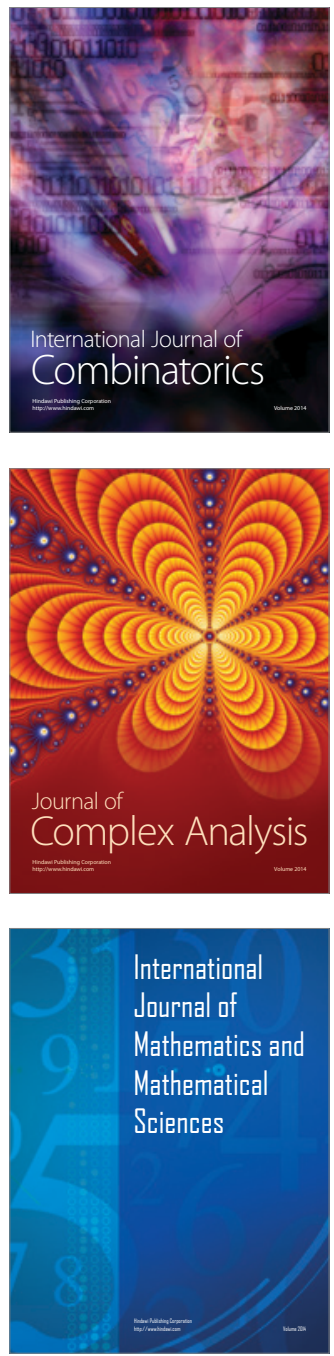
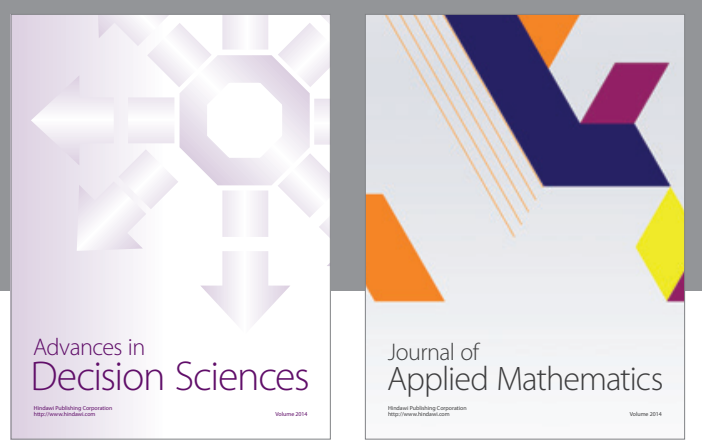

Algebra

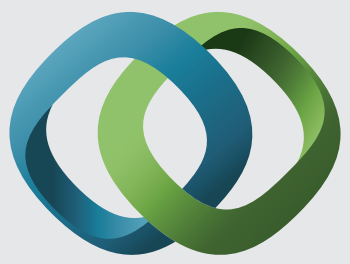

\section{Hindawi}

Submit your manuscripts at

http://www.hindawi.com
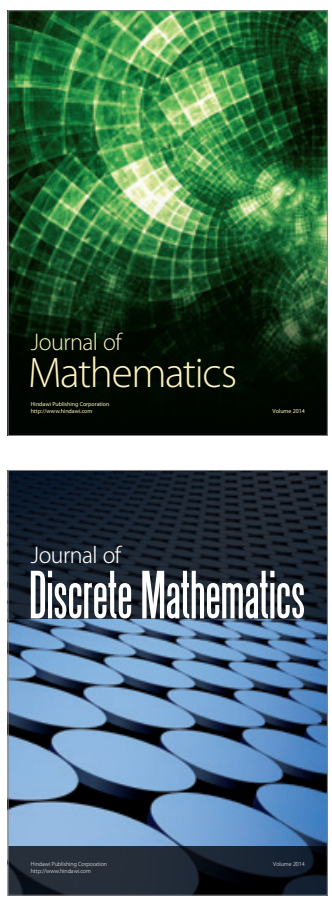

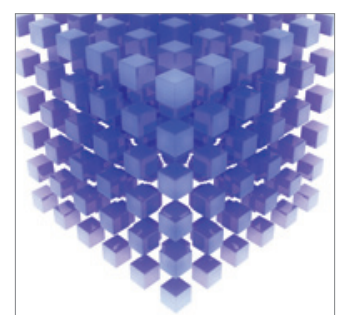

Mathematical Problems in Engineering
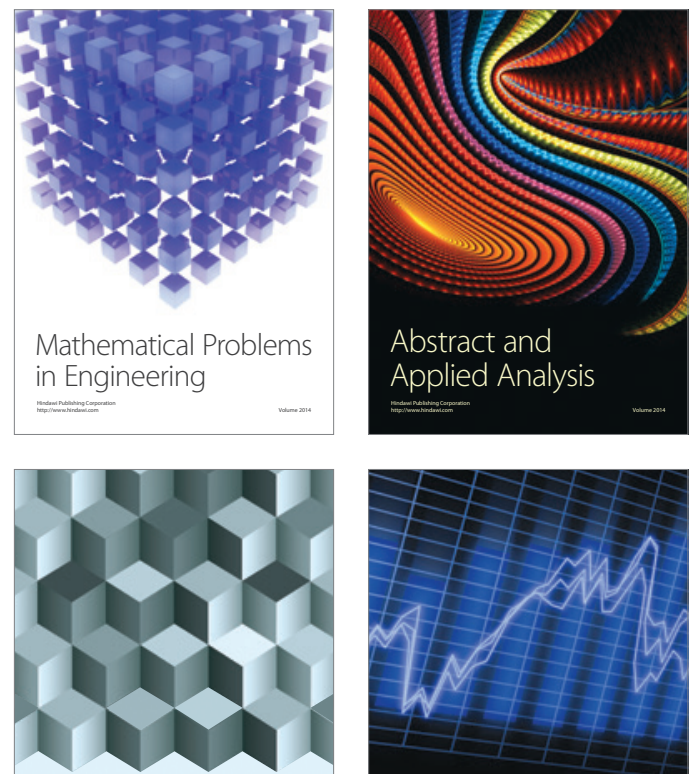

Journal of

Function Spaces

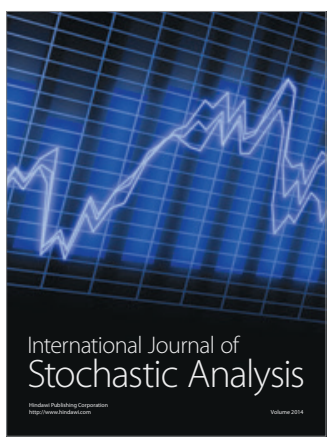

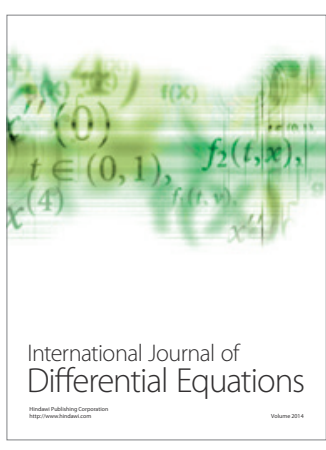
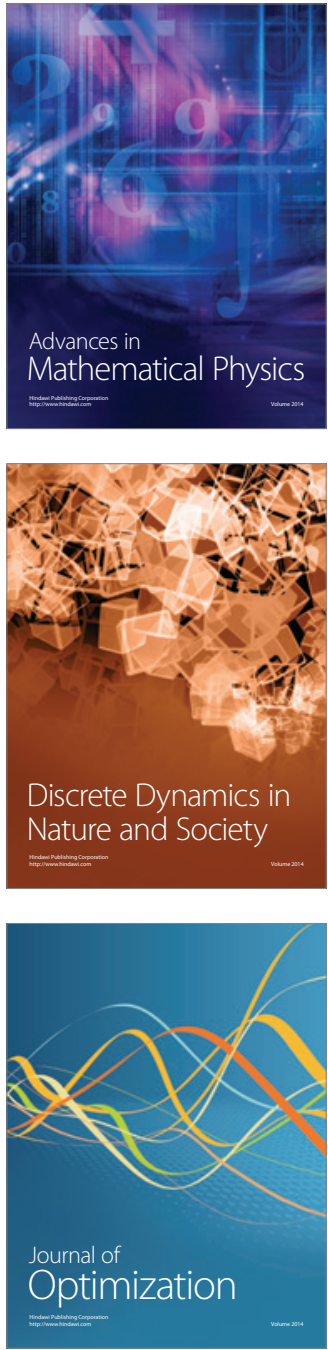